\title{
Neonatal arterial thromboembolism and limb loss following respiratory distress syndrome. Case report
}

\author{
Sung Shin Kim, $M D, P h D^{a}$, Ihl Sung Park, $M D^{b}$ and Hyun Sook Hong, $M D, P h D^{c}$
}

\begin{abstract}
Thromboembolic disease in newborn infants is a serious problem. The most important risk factors are iatrogenic factors, including indwelling umbilical catheters or central catheters. Other risk factors include asphyxia, dehydration, sepsis, cardiac disease, respiratory distress syndrome (RDS), disseminated intravascular coagulation, congenital thrombophilia (i.e. protein $\mathrm{C}$ or protein $\mathrm{S}$ deficiency), maternal diabetes mellitus, and passive transfer of maternal antiphospholipid antibodies. Neonates are more vulnerable to thrombosis than adults due to their reduced fibrinolytic capability. We describe a case of a 1-day-old female newborn with arterial thromboembolism in the lower leg without a central line catheter. The thromboembolismin in this case was associated with RDS. The infant underwent thrombolysis, anticoagulation therapy, and surgical thrombectomy. The leg of the infant was subsequently amputated below the knee. The case is described here with a brief review of relevant literatures.
\end{abstract}

Key words: amputation, arteries, respiratory distress syndrome, newborn, thromboembolism.

http:/ /dx.doi.org/10.5546/aap.2015.eng.e157

\section{INTRODUCTION}

Thromboembolism is a rare but severe disease in neonates that requires intensive care. The consequence could be devastating if timely and accurate treatment is not used. Various factors contribute to an increased risk of thromboembolic disease, including arterial and venous catheters, ${ }^{1}$ cardiac diseases, ${ }^{2}$ maternal diabetes, ${ }^{3,4}$ transfer of antiphospholipid antibodies, ${ }^{5}$ dehydration, sepsis, respiratory distress syndrome (RDS), disseminated intravascular coagulation, and

a. Department of Pediatrics, College of Medicine, Soonchunhyang University, Bucheon Hospital, Republic of Korea.

b. Department of Pediatrics, College of medicine, Soonchyuhyang University, Cheonnan Hospital, Republic of Korea.

c. Department of Radiology, College of Medicine, Soonchunhyang University, Bucheon Hospital, Republic of Korea.

E-mail Address:

Sung Shin Kim, M.D., Ph.D.: sungshin1201@gmail.com

Funding: this work was supported in part by the Soonchunhyang University Research Fund.

Conflict of interest: None.

Received: 10-9-2014

Accepted: 12-3-2014 asphyxia. ${ }^{4}$ We present a case of a neonate with an arterial thromboembolism who underwent amputation following RDS.

\section{CASE REPORT}

A female infant at $3100 \mathrm{~g}$ was born to a 30-year-old G2 P1 mother via a repeat cesarean section at 36 weeks gestation. Maternal prenatal screening test results were unremarkable. There was no history of hypertension, systemic lupus erythematosus, diabetes mellitus, renal disease, or thrombotic tendency. At 4 hours of life, she developed cyanosis and tachypnea. She was transferred to the nearest hospital. Chest x-ray showed ground glass opacities in both lungs with obliteration of cardiac borders. The patient was placed on ventilator care and given surfactant for RDS. She did not have any central catheters at this time. At 12 hours of age, her right leg was pale and cool $3 \mathrm{~cm}$ below the knee downward (Figure 1). No pulse was noted at the dorsal pedal artery of the right foot. White blood cell count was $16370 / \mathrm{mm}^{3}$ with $75 \%$ neutrophils. The hematocrit and C-reactive protein (CRP) were normal. The thrombophilia was evaluated. The protein $\mathrm{C}$, protein $\mathrm{S}$, and antithrombin levels were normal. Homozygous C677T polymorphism for methylenetetrahydrofolate reductase (MTHFR), heterozygous factor V Leiden, and G20210A mutation of the prothrombin gene was not

FIGURE 1. Limb ischemia involving the right leg

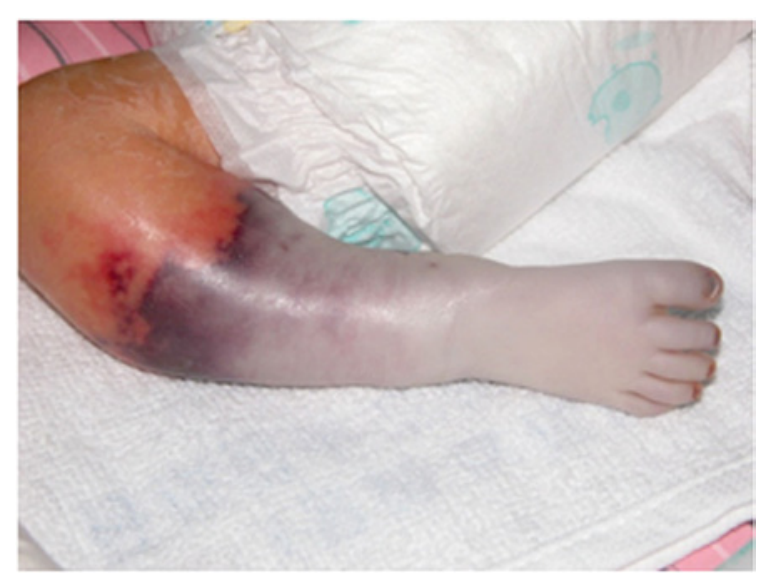


present. The platelet count was $96000 / \mathrm{mm}^{3}$. The prothrombin time was $15.6 \mathrm{sec}$ (normal, 10.1$15.9 \mathrm{sec}$ ). The activated partial thromboplastin time was $40.9 \mathrm{sec}$ (normal, 31.3-54.3 sec). The fibrinogen was $115 \mu \mathrm{g} / \mathrm{dL}$ (normal, 167$399 \mu \mathrm{g} / \mathrm{dL}$ ). The d-dimer level was $13536 \mu \mathrm{g} / \mathrm{L}$

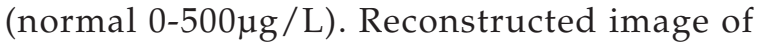
computed tomography (CT) angiogram failed to reveal the right internal iliac artery from iliac bifurcation (Figure 2). However, the right femoral artery was visualized to the mid-thigh level by recanalization. The distal, popliteal, anterior, and posterior tibial arteries were not observed either. The right femoral arterial flow had a lower peak, which was sluggish compared to that of the left femoral artery. Her venous flow was preserved (Figure 3). After recognizing vascular occlusion caused by arterial thromboembolism, the infant was immediately treated with thrombolysis, tissue plasminogen activator (tPA) at a dose of $0.5 \mathrm{mg} / \mathrm{kg} / \mathrm{hr}$ for 6 hours, and fresh frozen plasma. Unfractionated heparin $(20 \mathrm{U} / \mathrm{kg} / \mathrm{hr})$ was administered simultaneously to maintain low-dose anticoagulation.

FIGURE 2. Computed tomography (CT) angiogram reveals non-visualization of the right internal iliac artery from the iliac bifurcation. However, the right femoral artery was visualized down to the level of the mid-thigh following recanalization. The distal, popliteal, anterior, and posterior tibial arteries were not observed

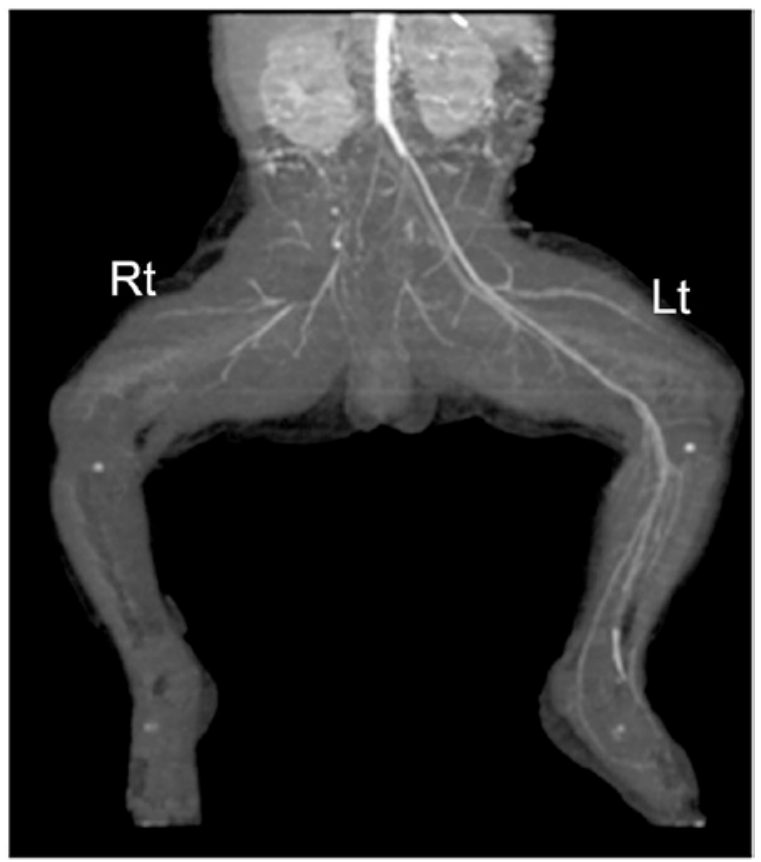

FIGURE 3. Doppler ultrasonography of the lower leg shows that the right femoral arterial flow had a lower peak and was sluggish compared with the wide left femoral artery. Venous flow was preserved. The right popliteal arterial flow was poorly defined (not shown). (A) Rt femoral artery,

(B) Lt femoral artery, (C) Rt femoral vein

A.

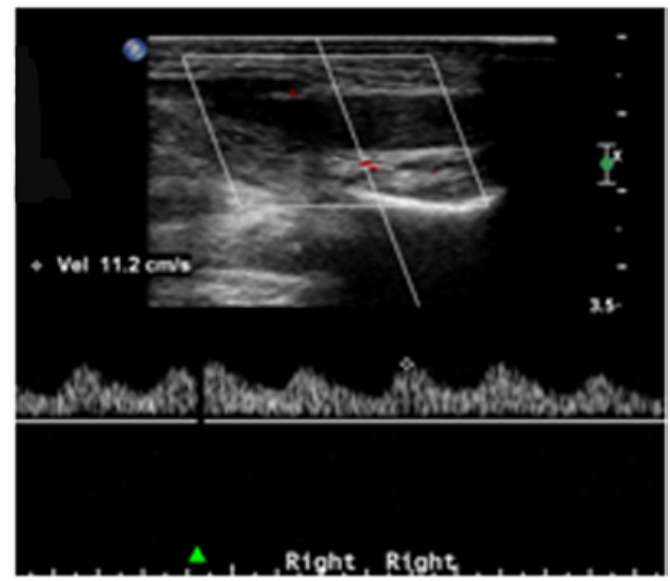

B.

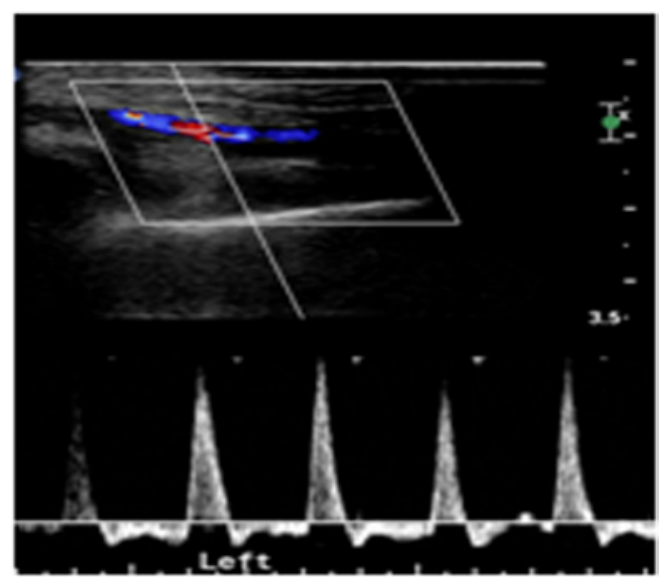

C.

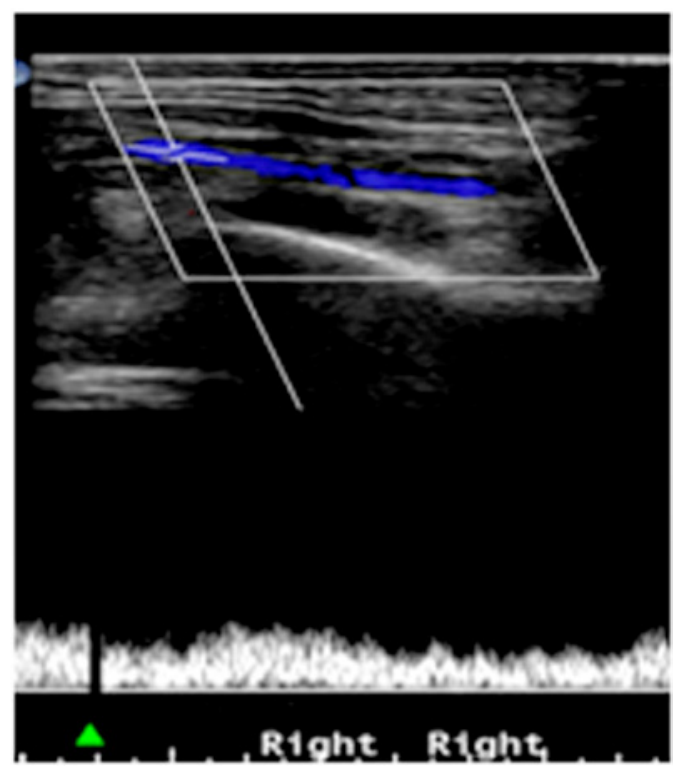


Before thrombolytic therapy, a head ultrasound was performed to determine whether a hemorrhage was present. Despite treatment, the infant showed no improvement in the viability of her lower leg. On the third day, a surgical thrombectomy was performed. Arterial flow was not restored to the lower leg. On postnatal day 20, the patient underwent below-the-knee amputation of the leg.

\section{DISCUSSION}

Infants are at risk of thromboembolism due to genetic and acquired factors. ${ }^{6} \mathrm{~A}$ hypofibrinolytic state (characterized by an undeveloped anticoagulation response with antithrombin, protein $C$, and protein $S$ deficiencies) increases the risk of thrombotic disease in neonates until equilibrium is restored. Inherited thrombophilia defects, including prothrombotic polymorphisms, factor $\mathrm{V}$ G1691A, factor II G2021A, and homozygous TT genotype of methylenetetrahydrofolate reductase C677T polymorphism, also increase the risk of thrombosis. ${ }^{7}$ In the case of thromboembolic event, we recommended to investigate the possibility of inherited thrombophilia by family counseling and long-term management. The risk can be exacerbated by other factors, such as diabetes mellitus, polycythemia, sepsis, heart disease, asphyxia, and RDS. ${ }^{8}$ Critically ill neonates are more susceptible to thromboembolism than healthy infants. ${ }^{9}$ Infants are more susceptible to lower limb arterial thromboembolism. Older children are more likely to have thromboembolism in the central nervous system. ${ }^{10}$ In the Canadian International Registry, 89\% of thrombosis cases were associated with intravascular catheters. ${ }^{11}$ Bruset al. ${ }^{12}$ reported that clotting, fibrinolysis, and kinin-kallikrein are concurrently activated in preterm infants with severe RDS causing altered coagulation. Fibrinolytic activity may provoke thromboembolism. A Doppler ultrasonography, $\mathrm{CT}$, magnetic resonance imaging (MRI) with or without magnetic resonance angiography (MRA), and venography can be used for diagnosis. Doppler ultrasound is the most frequently used technique to confirm a thromboembolism in critically ill newborns. Salvaging the limb depends on the length of time between the initial vascular event, the presentation, and the therapy. ${ }^{8}$ Supportive treatments include hydration, antibiotics, systemic or topical vasodilators, hyperbaric oxygen therapy, and systemic anticoagulation. ${ }^{13}$ Thromboembolism in children is rare compared to adults, rendering clinical trials challenging. Anticoagulation should use either low-molecular-weight heparin or unfractionated heparin. The suggested total duration of anticoagulation is between 6 weeks and 3 months. ${ }^{14}$ Thrombolytic therapy is only indicated when major vessel occlusion is critically compromising organs or limbs, or when the limb does not display fixed skin changes with established skin necrosis, or when the duration of the occlusion is short but ischemia is present. ${ }^{8}$ If thrombolysis is needed, tissue plasminogen activator and plasminogen supplementation with fresh frozen plasma are recommended. Concurrent heparin should be administered in prophylactic doses. ${ }^{15}$ Major intracranial hemorrhage may occur in up to $10 \%$ of preterm infants receiving the highest dose of tPA. ${ }^{15}$ Contraindications to thrombolysis include major surgery or hemorrhage, severe asphyxia, an invasive procedure, seizures, prematurity $<32$ weeks, septicemia, inability to maintain platelets $>50-100,000 / \mu \mathrm{L}$, and fibrinogen $>100$ $\mathrm{mg} / \mathrm{dL} .{ }^{18}$ Surgical intervention is suggested when thrombolytic therapy is contraindicated with organ or limb death pending. ${ }^{14}$ Amputation should be delayed for as long as possible as the demarcation line may be very distal to the original line of ischemia. Amputation should only be performed after fully considering future prosthetic limb application and the prevention of joint contracture. ${ }^{8}$

If a thrombotic condition is observed in a critically ill patient with or without risk factors, it should be managed quickly and thoroughly to salvage the organ or to prevent limb loss. To our knowledge, this is the first case report of an arterial thromboembolism leading to lower limb amputation following RDS in the absence of a central line catheter.

\section{Acknowledgement}

This work was supported in part by the Soonchunhyang University Research Fund.

\section{REFERENCES}

1. Boo NY, Wong NC, Zulkifli SS, Lye MS. Risk factors associated with umbilical vascular catheter-associated thrombosis in newborn infants. J Paediatr Child Health 1999;35(5):460-5.

2. Sträter R, Vielhaber $H$, Kassenböhmer R, von Kries R, et al. Genetic risk factors of thrombophilia in ischaemic childhood stroke of cardiac origin. A prospective ESPED survey. Eur J Pediatr 1999;158 (Suppl 3):S122-5.

3. Moazzam A, Riaz M, Brennen MD. Neonatal gangrene in an extremity of an infant of a diabetic mother. BJOG 
2003;110(1):74-6.

4. Nowak-Göttl U, Kosch A, Schlegel N. Neonatal thromboembolism. Semin Thromb Hemost 2003;29(2):22734.

5. Bhat MA, Kawoosa MS, Bhat JI, Ali SW. Antiphospholipid syndrome in a neonate. Pediatr Dermatol 2011;28(3):342-5.

6. Thornburg C, Pipe S. Neonatal thromboembolic emergencies. Semin Fetal Neonatal Med 2006;11(3):198-206.

7. Veldman A, Nold MF, Michel-Behnke I. Thrombosis in the critically ill neonate: incidence, diagnosis, and management. Vasc Health Risk Manag 2008;4(6):1337-48.

8. Arshad A, McCarthy MJ. Management of limb ischaemia in the neonate and infant. Eur J Vasc Endovasc Surg 2009;38(1):61-5.

9. Beardsley DS. Venous thromboembolism in the neonatal period. Semin Perinatol 2007;31(4):250-3.

10. Monagle P, Newall F, Barnes C, Savoia H, et al. Arterial thromboembolic disease: a single-centre case series study. J Paediatr Child Health 2008;44(1-2):28-32.

11. Schmidt B, Andrew M. Neonatal thrombosis: report of a prospective Canadian and international registry. Pediatrics 1995;96(5 Pt 1):939-43.

12. Brus F, Van Oeveren W, Okken A, Oetomo SB. Disease severity is correlated with plasma clotting and fibrinolytic and kinin-kallikrein activity in neonatal respiratory distress syndrome. Pediatr Res 1997;41(1):120-7.

13. Wiebers J, Purdy I, Lieber M, Milisavljevic V. Hyperbaric oxygen in treatment of neonatal arterial thromboembolism of lower extremities. J Perinatol 2006;26(12):777-9.

14. Monagle P, Chalmers E, Chan A, DeVeber G, et al. Antithrombotic therapy in neonates and children: American College of Chest Physicians Evidence-Based Clinical Practice Guidelines ( $8^{\text {th }}$ Edition). Chest 2008;133(6 Suppl):887s-968s.

15. Manco-Johnson MJ, Grabowski EF, Hellgreen M, Kemahli AS, et al. Recommendations for tPA thrombolysis in children. On behalf of the Scientific Subcommittee on Perinatal and Pediatric Thrombosis of the Scientific and Standardization Committee of the International Society of Thrombosis and Haemostasis. Thromb Haemost 2002;88(1):157-8. 